\title{
Preeclampsia y defecto cardiaco fetal: iexiste una asociación? Revisión de la evidencia
}

\author{
Isabel Silva-Ocas 1,2,a, José Gálvez-Olortegui 1,2, Tomás Gálvez-Olortegui1,3, Lida \\ Tavara-Valladolid $3,4,5$, Germán Fiestas-Plucker $2,4,6$, José Chaman-Castillo. $3,4,7$
}

${ }^{1}$ Unidad Generadora de Evidencias y Vigilancia Epidemiológica - Scientia Clinical and Epidemiological Research Institute, Trujillo-Perú. ${ }^{2}$ Facultad de Medicina Universidad Nacional de Trujillo, Trujillo-Perú. ${ }^{3}$ Escuela de Post Grado Universidad Privada Antenor Orrego, Trujillo-Perú. ${ }^{4}$ Unidad de Investigación Clínica - Scientia Clinical and Epidemiological Research Institute, Trujillo-Perú ${ }^{5}$ Departamento de Neonatología, Hospital Belén de Trujillo, Trujillo-Perú. ${ }^{6}$ Departamento de Ginecología y Obstetricia, Hospital Regional Docente de Trujillo, Trujillo-Perú. ${ }^{7}$ Departamento de Ginecología y Obstetricia del Hospital Belén de Trujillo, Trujillo-Perú.

\section{RESUMEN}

Introducción: La preeclampsia (PE) es un desorden multisistémico complejo causado por una angiogénesis anormal placentaria. La cardiopatía congénita (CC) es uno de los defectos estructurales más comunes en neonatos. Recientemente, diversos estudios han identificado un desequilibrio en los niveles de factores proangiogénicos y antiangiogénicos en sangre umbilical de neonatos con CC similares a los hallados en sangre de mujeres con PE, lo que sugiere una posible asociación. Objetivo: Revisar la evidencia científica disponible sobre la relación entre la PE y el desarrollo de CC en neonatos. Métodos: Se realizó una búsqueda en las bases de datos Scopus y Medline/Pubmed utilizando los términos "pre-eclampsia" y "congenital heart defects", se seleccionaron 4 artículos que relacionaban las variables PE y CC, los cuales fueron revisados a texto completo. Únicamente se encontraron trabajos de tipo observacional analítico (1 estudio de prevalencia, 1 estudio de casos y controles y 2 estudios de cohortes), publicados entre el 2014 y 2016. Resultados: La PE de inicio temprano (<34 semanas) fue el factor de riesgo más importante asociado al desarrollo de CC en neonatos. La severidad de un defecto cardiaco se asocia con la intensidad y el momento de inicio de los desequilibrios en los factores angiogénicos. Conclusión: Encontramos evidencia relevante de la asociación entre PE y CC. La condición hipertensiva y los cambios endoteliales condicionados por ésta, estarían relacionados con el aumento de riesgo para el desarrollo de la CC antes que la exposición a medicamentos antihipertensivos.

\section{PALABRAS CLAVES: Preeclampsia, hipertensión inducida en el embarazo, hipertensión, cardiopatía congénita, medicación antihipertensiva}

\section{SUMMARY}

Introduction: Preeclampsia (PE) is a complex multisystem disorder caused by an abnormal placental angiogenesis. Congenital heart disease (CHD) is one of the most common structural defects in newborn infants. Recently, several studies have identified an imbalance in the levels of proangiogenic and antiangiogenic factors in umbilical blood of newborn infants with CHD similar to those found in the blood of women with PE, suggesting a possible association. Objective: To review the available scientific evidence about the relationship between the PE and the development of CHD in newborn infants. Method: A search was conducted in Scopus and Medline/PubMed databases using the terms "pre-eclampsia" and "congenital heart defects". Four articles that linked PE and CHD were selected and reviewed in full text. Only analytical observational 
studies were found (1 prevalence study, 1 case-control study and 2 cohort studies), published between 2014 and 2016. Results: Early onset PE (<34 weeks) was the most important risk factor for the development of CHD in newborn infants. The severity of a heart defect is associated with the intensity and the onset time of imbalances in the angiogenic factors. Conclusion: We found relevant evidence on the possible association between PE and CHD. Hypertensive condition and endothelial changes conditioned by this seem to be linked to increased risk for the development of $\mathrm{CHD}$ instead of exposure to antihypertensive drugs.

\section{KEY WORDS: Pre-eclampsia, hypertension, pregnancy-induced, hypertension, congenital heart disease, antihypertensive medicate}

\section{INTRODUCCIÓN}

La preeclampsia (PE) es un desorden multisistémico que complica del 3 a $8 \%$ de los embarazos en los países occidentales y constituye la principal fuente de morbimortalidad materna a nivel mundial, llegando a presentar una prevalencia de $26 \%$ en Latinoamérica (1). La PE se define clínicamente como la aparición de proteinuria $\geq 300 \mathrm{mg} /$ día e hipertensión arterial (sistólica $\geq 140 \mathrm{mmHg}$ y/o diastólica $\geq 90 \mathrm{mmHg}$ ) a partir de las 20 semanas de gestación (2). Presenta una compleja fisiopatología, siendo la principal causa, la angiogénesis anormal de la placenta debido a una invasión defectuosa de las arterias espirales por las células del trofoblasto (3). Esta angiogenesis anormal se encuentra asociada a múltiples factores de riesgo.

Los factores de riesgo que se asocian a PE son hipertensión preexistente al embarazo, enfermedad renal, diabetes mellitus, obesidad, edad mayor a 35 años, embarazo múltiple, mola hidatiforme, antecedente de PE y anormalidades congénitas fetales (3). Así mismo la PE se asocia a diversas complicaciones fetales que incluyen la restricción del crecimiento intrauterino, prematuridad, defectos congénitos del sistema nervioso, digestivo, alteraciones hematológicas y recientemente se han descrito diversos tipos de defectos cardiacos (4).

La cardiopatía congénita (CC) es uno de los defectos estructurales más comunes en recién nacidos, reportándose prevalencias de 8 por 1000 nacidos vivos a nivel mundial y 9,1 en Sudamérica (5); siendo una condición que, aun recibiendo tratamiento, afecta la calidad de vida de las personas que la portan e incrementa los costos económicos y sociales tanto para la familia como para el Estado. A nivel molecular, diversos estudios han identificado un desequilibrio en los niveles de factores proangiogénicos como el factor de crecimiento endotelial vascular (VEGF), factor de crecimiento placentario (PIG) y el factor de crecimiento trofoblástico $\beta$ (TGF- $\beta$ ); y antiangiogénicos como el receptor de VEGF-1 o quinasa de tirosina similar al FMS soluble (sFlt-1), y la forma soluble de endoglina (sEng), en la sangre umbilical de neonatos con CC similar a los hallados en la sangre de mujeres con PE (6-8).
Estos hallazgos sugieren la existencia de una posible asociación entre PE y CC, por lo que el objetivo del presente estudio fue revisar la evidencia científica disponible sobre la relación entre la PE materna y el desarrollo de CC en el recién nacido.

\section{MATERIAL Y MÉTODO}

Estrategia de búsqueda y selección de estudios. Se realizó una búsqueda en las bases de datos Scopus y Medline/Pubmed utilizando los términos "pre-eclampsia" y "congenital heart defects", no se aplicaron filtros de idioma, tiempo, ni tipo de estudio; obteniendo 12 y 46 estudios respectivamente de los cuales se revisaron títulos y resúmenes. Se aplicaron como criterios de inclusión: estudios observacionales analíticos, ensayos clínicos o revisiones sistemáticas que relacionaran las variables PE y CC. No se realizó un intento de búsqueda de estudios no publicados.

Extracción y análisis de datos. El análisis de los estudios se realizó en dos fases, la primera fase consistió en la extracción de los siguientes datos: título, autores, revista y año de publicación; en la segunda fase, se extrajeron los datos relacionados al diseño, las variables, resultados, limitaciones y conclusiones de los estudios.

\section{RESULTADOS}

Solo 4 artículos cumplieron con los criterios de inclusión, los cuales fueron revisados a texto completo y ordenados siguiendo la pirámide de evidencia de las "6S" de DiCenso (9), y todos los trabajos seleccionados correspondieron al primer nivel de la pirámide: estudios primarios de tipo observacional analítico (1 estudio de prevalencia, 1 estudio de casos y controles y 2 estudios de cohortes) (Tabla I). Los trabajos revisados fueron publicados entre el año 2014 y 2016 . El objetivo de tres de los estudios fue determinar la relación de la PE materna como factor de riesgo para $C C$ en el neonato $(4,10,11)$, y sólo uno (12) relacionó la presencia de defectos cardiacos en el feto como factor de riesgo para el desarrollo de anomalías placentarias como la PE (Tabla II). 
Tabla I

TRABAJOS PUBLICADOS QUE ESTUDIAN LA RELACIÓN ENTRE LA PREECLAMPSIA Y EL DESARROLLO DE CARDIOPATÍA CONGÉNITA

\begin{tabular}{lcclll}
\hline Publicación & Autor & País & Tipo de estudio & Revista & Año \\
\hline $\begin{array}{l}\text { Possible common ae- } \\
\text { tiology behind maternal } \\
\text { preeclampsia and con- }\end{array}$ & $\begin{array}{c}\text { Brodwall K, } \\
\text { et al. }\end{array}$ & Canadá & Cohorte & Paediatric Perinatal & 2016 \\
Epidemiology &
\end{tabular}
genital heart defects in the child: a cardiovascular diseases in Norway Project Study (11)

Placenta-related complications in women carrying a foetus with congenital heart disease (12)

Maternal hypertensive disorders, antihypertensive medication usea, and the risk of birth defects: a case-control study (4)

Association between preeclampsia and congenital heart defects (10)

Ruiz A, España Cohorte retrospectiva

et al.

Van Gelder M, et al.

Noruega

Casos y controles
The Journal of Maternal-fetal and Neonatal Medicine

\footnotetext{
* Estudios ordenados por jerarquía de evidencia.
}

Auger $\mathrm{N}$,
et al.
quía de evidencia.

BJOG

\footnotetext{
* Estudios ordenados por jerarquía de evidencia.
}

Tabla II

\section{RESUMEN DE LOS HALLAZGOS DE LOS TRABAJOS ANALIZADOS}

\begin{tabular}{|c|c|c|c|c|c|}
\hline Estudios & Participantes & Variables & Resultados & Limitaciones & Conclusiones \\
\hline $\begin{array}{l}\text { Brodwall K. } \\
\text { et al } 2016 \\
(11)\end{array}$ & $\begin{array}{l}\text { Estudio de cohortes } \\
\text { de } 914.703 \text { recién } \\
\text { nacidos entre } 1994 \\
\text { y } 2009\end{array}$ & $\begin{array}{l}\text { EXP: } \\
\text { - PE de inicio } \\
\text { temprano } \\
\text { (<34semanas) } \\
\text { - PE de inicio } \\
\text { tardío } \\
\text { OUT: CC } \\
\text { severa } \\
\text { CoVar: año } \\
\text { y orden de } \\
\text { nacimiento, } \\
\text { edad materna } \\
\text { y diabetes pre- } \\
\text { gestacional }\end{array}$ & $\begin{array}{l}\text { Se mostró asociación } \\
\text { entre la PE de inicio } \\
\text { temprano y la CC se- } \\
\text { vera (RRa } 2,8 ; \text { IC95\% } \\
1,8-4,4) \text {. } \\
\text { El grupo de PE tardía } \\
\text { solo se asoció con la } \\
\text { forma severa }\end{array}$ & $\begin{array}{l}\text { No se identificó } \\
\text { obesidad materna } \\
\text { la cual se asocia a } \\
\text { PE y a CC. El regis- } \\
\text { tro de severidad e } \\
\text { inicio de PE inició } \\
\text { en } 1998 \text {. }\end{array}$ & $\begin{array}{l}\text { La asociación } \\
\text { es mayor para } \\
\text { la PE de inicio } \\
\text { temprano, es- } \\
\text { pecialmente con } \\
\text { VSDs. }\end{array}$ \\
\hline $\begin{array}{l}\text { Ruiz A, et } \\
\text { al. } 2015 \\
(12)\end{array}$ & $\begin{array}{l}\text { Estudio de una } \\
\text { cohorte retrospectiva } \\
\text { de } 279 \text { gestantes con } \\
\text { fetos con CC mayor } \\
\text { entre } 2013 \text { y } 2014 \text {. }\end{array}$ & $\begin{array}{l}\text { EXP: Fetos con } \\
\text { CC mayores } \\
\text { OUT: Com- } \\
\text { plicaciones } \\
\text { por anomalías }\end{array}$ & $\begin{array}{l}\text { Mayor incidencia } \\
\text { de PE en el grupo } \\
\text { de fetos con CC } \\
\text { comparada con fetos } \\
\text { normales }(5,7 \% \text { vs }\end{array}$ & $\begin{array}{l}\text { No se midieron los } \\
\text { índices Doppler de } \\
\text { la arteria uterina. No } \\
\text { se examinó la histo- } \\
\text { logía de las placen- } \\
\text { tas. No se reporta el } \\
\text { análisis de variables } \\
\text { confusoras. }\end{array}$ & $\begin{array}{l}\text { Riesgo mayor } \\
\text { de PE en mu- } \\
\text { jeres embara- } \\
\text { zadas de fetos } \\
\text { con CC. }\end{array}$ \\
\hline
\end{tabular}




\section{CONTINUACIÓN TABLA II}

\begin{tabular}{|c|c|c|c|c|c|}
\hline Estudios & Participantes & Variables & Resultados & Limitaciones & Conclusiones \\
\hline & & $\begin{array}{l}\text { placentarias } \\
\text { (PE, eclamp- } \\
\text { sia, Síndrome } \\
\text { HELLP, óbito } \\
\text { fetal, RCIU). }\end{array}$ & $\begin{array}{l}1,2 \% ; \mathrm{p}<0,0001)(\mathrm{OR} \\
5,96 ; \text { IC95\% 3,19- } \\
10,54) \text {. Diferencia con } \\
\text { el grupo VADs }(7,7 \% \\
\text { vs } 1,2 \% ; \mathrm{p}<0,0001) \text { y } \\
\text { anomalías conotrun- } \\
\text { cales }(5,8 \% \text { vs } 1,2 \% \text {; } \\
p<0,0003) \text {. }\end{array}$ & & \\
\hline $\begin{array}{l}\text { Van Gelder } \\
\text { M, et al. } \\
2014 \text { (4) }\end{array}$ & $\begin{array}{l}\text { Estudio de casos y } \\
\text { controles de } 12.821 \\
\text { recién nacidos entre } \\
1998 \text { y } 2010 .\end{array}$ & $\begin{array}{l}\text { EXP: } \\
\text { - Enfermedad } \\
\text { hipertensiva: } \\
\text { HTAC, HTAC + } \\
\text { PE, hiperten- } \\
\text { sión gestacio- } \\
\text { nal, PE) } \\
\text { - Consumo } \\
\text { de fármacos } \\
\text { antiadrenér- } \\
\text { gicos, IECAs, } \\
\text { ARA-II, calcio } \\
\text { antagonistas, } \\
\text { vasodilatado- } \\
\text { res } \\
\text { OUT: Defectos } \\
\text { congénitos: CC }\end{array}$ & $\begin{array}{l}\text { Mayor riesgo de VSDs } \\
\text { (ORa: 1,5; IC95\% } \\
\text { 1,1-2,2) y defectos } \\
\text { del corazón izquierdo } \\
\text { (ORa 1,6, IC95\% 1,0- } \\
2,6) \text { en recién nacidos } \\
\text { de madres con PE sin } \\
\text { tratamiento farmaco- } \\
\text { lógico. El riesgo de } \\
\text { VSDs fue mayor en } \\
\text { mujeres con manejo } \\
\text { no farmacológico } \\
\text { (ORa 3,9; IC95\% 1,3- } \\
\text { 11,7) y farmacológico } \\
\text { (ORa 3,9; IC95\% 1,3- } \\
\text { 10,7) de PE sobre- } \\
\text { agregada a HTAC. }\end{array}$ & $\begin{array}{l}\text { No se tuvo infor- } \\
\text { mación sobre la } \\
\text { severidad de la } \\
\text { enfermedad hiper- } \\
\text { tensiva. }\end{array}$ & $\begin{array}{l}\text { Los resulta- } \\
\text { dos apoyan la } \\
\text { hipótesis de } \\
\text { que los cambios } \\
\text { fisiológicos } \\
\text { tempranos de } \\
\text { la hipertensión } \\
\text { gestacional y PE } \\
\text { puede jugar un } \\
\text { rol en la etiolo- } \\
\text { gía de defectos } \\
\text { congénitos } \\
\text { mayores como } \\
\text { la CC. }\end{array}$ \\
\hline $\begin{array}{l}\text { Auger N, } \\
\text { et al. } 2015 \\
\text { (10) }\end{array}$ & $\begin{array}{l}\text { Estudio transver- } \\
\text { sal analítico de } \\
1.942 .072 \text { recién } \\
\text { nacidos entre } 1989 \\
\text { y } 2012 \text {. }\end{array}$ & $\begin{array}{l}\text { EXP: } \\
\text { - PE de inicio } \\
\text { temprano } \\
\text { - PE de inicio } \\
\text { tardío } \\
\text { OUT: } \\
\text { - CC críticas } \\
\text { - CC no críticas } \\
\text { CoVar: Comor- } \\
\text { bilidad y edad } \\
\text { materna, pari- } \\
\text { dad, nacimien- } \\
\text { to múltiple y } \\
\text { nivel socioeco- } \\
\text { nómico. }\end{array}$ & $\begin{array}{l}\text { Prevalencia de CC } \\
\text { en hijos de madres } \\
\text { con PE fue } 16,7 / 1 \\
000 \text { vs } 8,6 / 1 \text { 000 en } \\
\text { los niños de madres } \\
\text { sin PE. Los niños de } \\
\text { madres con PE tem- } \\
\text { prana tuvieron mayor } \\
\text { prevalencia de CC crí- } \\
\text { ticas (336,4/100.000; } \\
\text { RP: } 2,78 ; \text { IC95\% } \\
1,71-4,50 ; \text { DP: } \\
249,6 / 100.000 \text {, } \\
\text { (IC } 89,7-409,6) \\
\text { y CC no críticas } \\
\text { (7306,9/100.000; RP: } \\
5,55 ; \text { IC 4,98-6,19; } \\
\text { DP: } 6089,2 / 100.000 ; \\
\text { IC 5350,0-6828,3). }\end{array}$ & $\begin{array}{l}\text { No se obtuvieron } \\
\text { datos de CC en } \\
\text { óbitos, abortos y } \\
\text { embarazos inte- } \\
\text { rrumpidos, ni de } \\
\text { consumo de medi- } \\
\text { camento antihiper- } \\
\text { tensivo o consumo } \\
\text { de folato. Evaluaron } \\
\text { solo diagnósticos } \\
\text { de alta. }\end{array}$ & $\begin{array}{l}\text { La PE estuvo } \\
\text { asociada sig- } \\
\text { nificativamente } \\
\text { con CC no } \\
\text { crítica. La PE de } \\
\text { inicio temprano } \\
\text { estuvo asociada } \\
\text { a CC críticas. El } \\
\text { riesgo absoluto } \\
\text { de CC fue bajo. }\end{array}$ \\
\hline
\end{tabular}

PE: Preeclampsia, CC: Cardiopatía congénita, RCIU: Restricción del crecimiento intrauterino HTAC: Hipertensión arterial crónica, HELLP: Hemólisis, enzimas hepáticas elevadas y plaquetopenia. IECAs: Inhibidores de la enzima convertidora de angiotensina, ARA-II: Antagonista de receptores de angiotensina II. VSDs: Defectos del tabique ventricular, ASD: Defectos del tabique auricular, VADs: Defectos aurículo ventriculares. Exp: Exposición, Out: Outcome, CoVar: Covariables. ORa: OR ajustado, RRa: RR ajustado. RP: razón de prevalencias. DP: diferencia de prevalencias. 


\section{DISCUSIÓN}

Inicio de los síntomas de la PE. En un embarazo normotenso, los niveles de sFlt-1 se estabilizan alrededor de la mitad del embarazo, seguido de un incremento a partir de la semana 33, incremento que se corresponde con la fase en la que hay una disminución de los niveles de PIGF libre (8). En el caso de la PE, este proceso de variación de los niveles de sFlt-1 y PIGF parecen ocurrir mucho antes, lo que conllevaría a la disfunción endotelial $(6,8)$. La PE de inicio temprano también se conoce como PE placentaria porque su etiología se asocia con una placentación anormal bajo condiciones hipóxicas con niveles elevados de sFlt-1 y bajos niveles de PIGF, y se cree que la sobre expresión de la sEng y sFIt-1 inhibe el remodelamiento de la arteria espiral en la interfase placentaria, es decir este proceso se da desde el inicio del embarazo $(13,14)$. En contraste la PE de inicio tardío, o PE materna, no se asocian a una placentación anormal, sino que se considera una respuesta descompensada al estrés oxidativo generado por una disfunción endotelial placentaria que hace a la mujer susceptible al daño microvascular y alteración en el desarrollo de estructuras cardiovasculares fetales $(6,15)$.

Se ha reportado que la PE de inicio temprano se asocia con defectos que involucran casi la totalidad de las estructuras cardiacas, mientras que la PE de inicio tardío se asocia con defectos septales, considerando que el septo presenta un desarrollo más prolongado y podría verse afectado por la inadecuada función placentaria tardía $(10,12)$.

Auger y cols (10) y Brodwall y cols (11) reportaron que la PE de inicio temprano (<34 semanas) fue el factor de riesgo más importante para el desarrollo de CC en neonatos. Auger y cols reportaron una asociación significativa entre la PE de inicio temprano y el desarrollo de CC de tipo crítico con una razón de prevalencia de 2,78 (IC95\% 1,71$4,50)$, una diferencia de prevalencia de 249,6 en 100.000 y un RR ajustado de 2,8 (IC95\% 1,8-4,4). Por otro lado, Brodwall y cols reportaron que la PE de inicio temprano tanto leve como severa estuvieron asociados con un incremento de riesgo de CC con un RR de 15,5 (IC95\% 7,9-30,4) y un RR ajustado de 13,5 (IC95\% 6,8-26,8), especialmente con la doble salida del ventrículo derecho (double outlet right ventricle, DORV), síndrome de corazón derecho hipoplásico (hypoplastic right heart síndrome, HRHS), coartación de la aorta y con defecto del tabique auriculoventricular (atrioventricular septal defect, AVSD); esta última, presente en más del $25 \%$ de los casos de PE de inicio temprano severo. Solo se encontró un incremento de riesgo de CC en el grupo de PE severa tardía con un RR de 1,7 (IC95\% 1,2-2,4). Las 3 CC más comunes fueron los defectos de la arteria pulmonar y aorta, de válvula y de tabique.
De la severidad de la CC. Brodwall y cols (11) limitaron su estudio a casos de CC severa, entre los que se incluyeron: heterotaxia, transposición de grandes vasos, tetralogía de Fallot, DORV, otros defectos conotruncales (estenosis aórtica supravalvular, defecto del tabique ventricular (ventricular septal defects, VSD), tronco arterial común, arco aórtico interrumpido), AVSD, retorno venoso pulmonar anómalo, hipoplasia del corazón izquierdo, estenosis valvular aórtica, HRHS, anomalía de Ebstein. Por otro lado, Auger y cols (10) clasificaron a las CC en críticas y no críticas, incluyendo en el primer grupo: tetralogía de Fallot, transposición de grandes vasos, tronco arterial, hipoplasia del corazón izquierdo, coartación aórtica. Los casos de ductus arteriovenoso permanente solo fueron considerados en los neonatos a término. En ninguno de los trabajos se especificó los criterios para considerar una CC de tipo severo o crítico, sin embargo, se debe tener en cuenta que la severidad de un defecto cardiaco se asocia con la intensidad y el momento de inicio de los desequilibrios en los factores angiogénicos.

Se ha reportado un incremento de los niveles de VEGF-A en los casos de anomalías severas de la formación del corazón, tales como: sobreproducción de trabéculas, tabicación ventricular defectuosa y remodelamiento de los tractos de eyección. Además, se identificaron niveles doblemente incrementados de sFlt-1 en sangre umbilical de neonatos con CC comparado con controles (7).

Medicamentos antihipertensivos. Los medicamentos de primera línea indicados para el manejo de la hipertensión en la preeclampsia son metildopa y labetalol, en casos severos se recomienda el uso de nifedipino e hidralazina $(2,16)$. Fármacos como los inhibidores de la enzima convertidora de angiotensina (IECAs), los antagonistas de los receptores de angiotensina II (ARA-II) y los inhibidores de renina están contraindicados en gestantes por haberse demostrado su potencial teratogénico $(2,17)$. Debido a que el $50 \%$ de los embarazo no son planificados, tampoco se recomienda el consumo de estos fármacos en mujeres en edad reproductiva, en el caso de que se haya iniciado el manejo con estos medicamentos se debe modificar la pauta cuando se haga el diagnóstico de embarazo (2).

De los estudios revisados, Van Gelder y cols (4) evaluaron la relación entre condiciones hipertensivas maternas (hipertensión crónica, PE sobreagregada a hipertensión crónica, hipertensión gestacional, PE y síndrome de HELLP) y el desarrollo de defectos congénitos de diversos tipos entre los que se incluye la $\mathrm{CC}$; siendo el único estudio que evaluó el efecto del consumo de medicamentos antihipertensivos, y reportando que entre las mujeres con manejo no farmacológico y farmacológico para PE sobreagregada a 
hipertensión crónica, el riesgo de VSD fue mayor que en las mujeres sanas con un OR ajustado de 3,9 (IC95\% 1,3-11,7) y OR ajustado de 3,7 (IC95\% 1,3$10,7)$ respectivamente; sin embargo no se reportó diferencias entre los grupos con y sin tratamiento farmacológico. A pesar del número pequeño de casos de defectos del tabique auricular (auricular septum defects, ASD) secundum $(n=4)$, se observó un riesgo incrementado para esta patología en el grupo sin tratamiento farmacológico con un OR ajustado de 6,5 (IC95\% 1,8-23,7).

Por otro lado, el grupo que recibió tratamiento farmacológico presentó un incremento de riesgo para presentar defectos del lado izquierdo con un OR ajustado de 3,8 (IC95\% 1,1-13,2), Li y cols (18) reportaron un incremento de riesgo no significativo para el desarrollo de CC en neonatos de mujeres con hipertensión, tratadas con IECAs durante el primer trimestre del embarazo en comparación con controles normales con un OR 1,54 (IC95\% 0,90$2,62)$. Este incremento de riesgo no se identificó cuando se compararon con mujeres hipertensas que no recibieron IECAs ni ningún otro tratamiento antihipertensivo durante el primer trimestre, aunque la hipertensión por sí misma se asoció a un incremento de riesgo de $41 \%$ para el desarrollo de CC.

Los hallazgos de los trabajos referidos refuerzan la hipótesis de que la condición hipertensiva y los cambios endoteliales condicionados por ésta, estarían relacionados con el aumento de riesgo para el desarrollo de la CC antes que la exposición a medicamentos antihipertensivos.

Defectos cardiacos fetales como factor de riesgo para el desarrollo de PE. El desarrollo del corazón se inicia en la tercera semana gestacional y para la octava semana, las estructuras mayores del corazón (cámaras, tractos de salida y almohadillas endocárdicas) están formadas (19). Posteriormente la maduración cardiaca continúa, hasta completar la formación de los tabiques auricular y ventricular, la división de la arteria pulmonar y aorta, y el desarrollo valvular $(20,21)$. Se ha postulado que la invasión trofoblástica se daría en dos fases conocidas como "oleadas", la primera (invasión superficial) se iniciaría hacia la semana 10, y la segunda (invasión profunda) hacia la semana 14-15 (22). Es decir, antes de que se inicie la invasión trofoblástica ya se habría desencadenado el desequilibrio de los factores angiogénicos condicionado por el defecto cardiaco del embrión, el cual podría alterar el desarrollo de la vasculatura placentaria y condicionar el desarrollo de la PE. Tanto en mujeres con PE como en madres de fetos con defectos conotruncales y de válvulas cardiacas se han identificado niveles séricos disminuidos de PIGF durante las semanas 11 a 13 de gestación (7).

El estudio de Ruiz y cols (12), fue el único que asoció la presencia de defectos cardiacos mayores en fetos (defectos de las válvulas auriculoventriculares, anomalías conotruncales y obstrucciones de salida del ventrículo izquierdo) con la incidencia de complicaciones por anomalías placentarias (PE, eclampsia, síndrome de HELLP, óbito fetal y restricción del crecimiento intrauterino). Ellos reportaron una incidencia significativamente mayor de PE en el grupo de embarazadas de fetos con defectos cardiacos comparada con la población normal $(5,7 \%$ vs $1,2 \% ; p<0,0001)$, así como un OR 5,96 (IC95\% 3,19-10,54). Se excluyeron anomalías cardiacas estructurales como tumores o arritmias, defectos cardiacos menores o anomalías venosas sistémicas.

Por lo antes mencionado planteamos la necesidad de elaborar un protocolo de seguimiento a las gestantes con preeclampsia más allá de lo registrado en las historias clínicas o en los sistemas informáticos perinatales de cada país, a fin de conocer la magnitud del efecto y las consecuencias clínicas que conlleva esta patología; además esto permitiría la realización de nuevos estudios en Latinoamérica.

\section{CONCLUSIÓN}

Los hallazgos de los trabajos observacionales respaldados con los datos de alteración de los niveles de factores angiogénicos plantean una evidencia relevante sobre la posible asociación entre PE y CC.

\section{REFERENCIAS}

1. Khan K, Wojdyla D, Say L, Gülmezoglu AM, Van Look PF. WHO analysis of causes of maternal death: a systematic review. Lancet 2006;367(9516):1066-74.

2. American College of Obstetricians and Gynecologists; Task Force on Hypertension in Pregnancy . Hypertension in pregnancy. Report of the American College of Obstetricians and Gynecologists' Task Force on Hypertension in Pregnancy. Obstet Gynecol 2013;122(5):1122-31.

3. Uzan J, Carbonnel M, Piconne O, Asmar R, Ayoubi JM. Pre-eclampsia: Pathophysiology, diagnosis, and management. Vasc Health Risk Manag 2011;7(1):46774.

4. Van Gelder M, Van Bennekom C, Louik C, Werler M, Roeleveld N, Mitchell A. Maternal hypertensive disorders, antihypertensive medication use, and the risk of birth defects: a case-control study. An Int J Obstet Gynaecol 2015;122(7):1002-9.

5. Van der Linde D, Konings E, Slager M, Witsenburg M, Helbing W, Takkenberg JJM, et al. Birth Prevalence of Congenital Heart Disease Worldwide. J Am Coll Cardiol 2011;58(21):2241-7.

6. Phipps E, Prasanna D, Brima W, Jim B. Preeclampsia: Updates in Pathogenesis, Definitions, and Guidelines. Clin J Am Soc Nephrol 2016;11(6):1102-13.

7. Llurba E, Sánchez O, Ferrer Q, Nicolaides K, Ruíz A, Domínguez $\mathrm{C}$, et al. Maternal and foetal angiogenic imbalance in congenital heart defects. Eur Heart $\mathrm{J}$ 2014;35(11):701-7. 
8. Sliwa K, Mebazaa A. Possible joint pathways of early pre-eclampsia and congenital heart defects via angiogenic imbalance and potential evidence for cardioplacental syndrome. Eur Heart J 2014;35(11):680-2.

9. DiCenso A, Bayley L, Haynes RB. Accessing preappraised evidence: fine-tuning the $5 \mathrm{~S}$ model into a 6S model. Evid Based Nurs 2009;12(4):99-101.

10. Auger N, Fraser W, Healy-Profitós J, Arbour L. Association Between Preeclampsia and Congenital Heart Defects. JAMA 2015;314(15):1588-98.

11. Brodwall K, Leirgul E, Greve G, Vollset S, Holmstrøm $\mathrm{H}$, Tell $\mathrm{G}$, et al. Possible Common Aetiology behind Maternal Preeclampsia and Congenital Heart Defects in the Child: A Cardiovascular Diseases in Norway Project Study. Paediatr Perinat Epidemiol 2016;30(1):76-85.

12. Ruiz A, Ferrer Q, Sánchez O, Ribera I, Arévalo S, Alomar $\mathrm{O}$, et al. Placenta-related complications in women carrying a foetus with congenital heart disease. $\mathrm{J} \mathrm{Ma-}$ tern Fetal Neonatal Med 2016;29(20):1-5.

13. Steegers E, Von Dadelszen P, Duvekot J, Pijnenborg R. Pre-eclampsia. Lancet 2010;376(9741):631-44.

14. Noori M, Donald A, Angelakopoulou A, Hingorani A, Williams D. Prospective study of placental angiogenic factors and maternal vascular function before and after preeclampsia and gestational hypertension. Circulation 2010;122(5):478-87.
15. Von Dadelszen P, Magge L. Preeclampsia: An update. Curr Hupertens Rep 2014;16(8):454.

16. van den Bosch AE, Ruys TP, Roos-Hesselink JW. Use and impact of cardiac medication during pregnancy. Future Cardiol 2015;11(1):89-100.

17. Sato $R$, Ikuma $M$, Takagi $K$, Yamagishi $Y$, Asano J, Matsunaga $\mathrm{Y}$, et al. Exposure of Drugs for Hypertension, Diabetes, and Autoimmune Disease During Pregnancy and Perinatal Outcomes. Medicine 2015:94(1):e386.

18. Li D-K, Yang C, Andrade S, Tavares V, Ferber JR. Maternal exposure to angiotensin converting enzyme inhibitors in the first trimester and risk of malformations in offspring: a retrospective cohort study. BMJ 2011;343:d5931.

19. Srivastava D. Making or breaking the heart: From lineage determination to morphogenesis. Cell 2006;126(6):1037-48.

20. Bruneau BG. The developmental genetics of congenital heart disease. Nature 2008;451(7181):943-8.

21. Dhanantwari $P$, Lee E, Krishnan A, Samtani R, Yamada S, Anderson S, et al. Human cardiac development in the first trimester: A high-resolution magnetic resonance imaging and episcopic fluorescence image capture atlas. Circulation 2009;120(4):343-51.

22. Lyall F. The Human Placental Bed Revisited. Placenta 2002;23(8-9):555-62. 